\title{
Relationship between Happiness and Gender Inequality Index: Canonical Commonality Analysis
}

\author{
Özlem Yorulmaz ${ }^{1}$ \\ ${ }^{1}$ Statistics Division, Department of Econometrics, Faculty of Economics, İstanbul University, Istanbul, Turkey \\ Correspondence: Özlem Yorulmaz, Assistant Professor, Statistics Division, Department of Econometrics, Faculty of \\ Economics, İstanbul University, Istanbul, Turkey.
}

Received: February 11, 2016

Accepted: February 24, 2016

Online Published: March 7, 2016

doi:10.5430/rwe.v7n1p11

URL: http://dx.doi.org/10.5430/rwe.v7n1p11

\begin{abstract}
Happiness is an important subject for policy makers in today's world as improving happiness has been recognized as important for increasing the gross national product (GNP) of a country. Although the relationship between gender inequality and happiness has been investigated in previous studies, gender inequality has not been considered from the perspective of reproductive health, empowerment, and economic status. This study considers these perspectives at the country level and evaluates the relationship between gender inequality and happiness using the sub-indicators of Gender Inequality Index (GII) and Happy Planet Index (HPI) for canonical commonality analysis of 150 countries. Findings reveal a significant correlation between HPI and GII and that maternal mortality rate has a more unique effect on the canonical covariate compared to economic status and empowering women.
\end{abstract}

Keywords: canonical commonality analysis, gender inequality index, happy planet index

\section{Introduction}

Happiness is increasingly considered an important and useful way to guide and measure the effectiveness of public policy (Cho, 2015). Alongside the economic performance of a country, social, cultural, political, and environmental factors may have effects on the happiness of people. Therefore, the happiness of a country may be a good indicator of the distribution of economic resources and the policy and environmental conditions for some countries. Currently, policy makers are discussing whether improving happiness is as crucial as increasing gross national product (GNP) for developing countries and the concept of gross national happiness (GHN) had been recognized globally. A new economy is needed to produce good living conditions that do not negatively impact the Earth. This may require a radical shift from the current system so that twenty-first century politicians, non-governmental organizations, and academics look beyond economic growth to understand progress (NEF, 2012).

The relationship between happiness and economic factors has been investigated in many studies. The findings reveal that the relationship is not the same within nations and across nations. Clark and Oswald's (1994) study with Diener \& Seligman (2004) emphasized that income has no significant effect on happiness. However, Veenhoven (1994) and Diener, Sandvik, Seidlitz, \& Diener, (1993) found a significant correlation between income and happiness within countries. Conversely, Kahneman \& Deaton (2010) found that rather than economic factors, social relationships have a greater effect on happiness. On the other hand, Huppert \& Whittington (2003) found a significant relationship between unemployment and well-being.

Researchers generally combine subjective surveys with objective data (i.e., on education, health, and income) to measure happiness. The New Economic Foundation (NEF) introduced an index of human well-being called the Happy Planet Index (HPI) in 2006. The index is based on three indicators-well-being (WE), life expectancy (LE), and ecological footprint (FT) - that reveal which countries are the most efficient at producing long, happy lives for their inhabitants whilst maintaining the conditions for future generations to do the same (NEF, 2010). HPI shows the relative efficiency with which nations deliver human well-being (Marks et al. 2006).

Life satisfaction can be evaluated through data from surveys. A question called the 'Ladder of Life' from the Gallup World Poll is often used. This asks respondents to imagine a ladder, where 0 represents the worst possible life and 10 the best possible life, and report the step of the ladder on which they are currently standing (NEF, 2010). Data for the other component of this measure, life expectancy, is provided in United Nations Human Development Programme 
(UNDP) reports. The ecological footprint, which is a metric of human demand on nature, corresponds to the assessed average productive capacity of a hectare of land. Considering the aforementioned three indices, HPI is calculated as follows:

$$
H P I \approx \frac{\text { well-being } x \text { life expectancy }}{\text { Ecological Footprint }}
$$

Because of awareness of the insufficiency of economic parameters for measuring the development of countries, a new index called the Human Development Index (HDI) was introduced in the 1990 UNDP report. However, the UNDP report indicates drawbacks to human development from gender inequality and mentions the negative effects of discrimination against women in the areas of education, health, and politics. To provide deeper insights regarding the gender distinctness of human development, the Gender Inequality Index (GII) was introduced in the 2010 UNDP report. The GII is based on three measures: reproductive health, which is determined by maternal mortality rates and adolescent birth rates; empowerment, which is measured by the proportion of seats in parliament held by women; and the ratio of females and males with at least a secondary school education. The third aspect is related to economic status and is represented by the labour-force participation ratio of female and male populations. Higher GII values correspond to more distinction between females and males and hence more loss to human development (UNDP, 2014).

The capability approach (Sen, 2005) is focused on what individuals are able to do (i.e., activities such as working, political action and literacy) and underlies the concept of human development. Sen's study also investigated the relationship between the gender gap and human development. Other studies have considered the effects of women's social status, education, and health. Happiness and gender inequality studies have mainly attempted determine if men or women are happier and if educational level, marital status, occupational status, and age affect their happiness (Cesare \& Amori, 2006; Easterlin, 2001). The studies of Blanchflower \& Oswald (2004) and Praag \& Ferrer-i-Carbonell (2008) with Tiefenbach \& Kohlbacher (2013) found that women are happier compared to men. Tiefenbach \& Kohlbacher (2013) also found that age affects happiness for men but not for women.

To the best of our knowledge, previous studies have not considered the relationship between happiness and gender inequality from the viewpoints of reproductive health, empowerment, and economic conditions. This study investigates the relationship between the sub-indicators of HPI and the sub-indicators of GII for 150 countries using canonical commonality analysis. In addition to measuring the relationship between two multivariate data sets, canonical commonality analysis clarifies the role of multicollinearity by partitioning the canonical variate and obtaining unique and common effects.

The rest of the paper is organized into four sections. Section 2 explains canonical commonality analysis. Section 3 presents data and findings, and Section 4 provides a conclusion.

\section{Methodology}

Firstly, the relationship between the sub-indices of HPI and the sub-indices of GII was investigated with canonical correlation analysis (CCA). The analysis was developed by Hotelling (1936) and measures the relationship between two multivariate data sets. CCA reveals the common structure of two multivariate data sets by constructing linear combinations of variables from each data set, called canonical variates, so that the correlation between the two canonical variates is maximized (McGarigal et al., 2000). Canonical variates are formed from the weights of original variables, which are calculated from their contribution to the variance of the relevant canonical variate. The first pair of variates defines the highest correlation in the analysis. The dimensionality in CCA is equal to or less than the smallest dimensionality of the two data sets.

The purpose of CCA is to find two bases in which the correlation between the projections of the variables onto these basis vectors are mutually maximized (Borga, 2001). Consider that for two data sets $\mathrm{X}$ and $\mathrm{Y}$, the linear combinations of $\mathrm{X}$ and $\mathrm{Y}$ correspond to $\mathrm{x}$ and $\mathrm{y}$;

$$
x=X^{\prime} \hat{w}_{x} \quad y=Y^{\prime} \hat{w}_{y}
$$

A basis that corresponds to the largest canonical correlation can be found by maximizing the function below. 


$$
\begin{aligned}
\rho & =\frac{E(x y)}{\sqrt{E\left(x^{2}\right) E\left(y^{2}\right)}}=\frac{E\left(\hat{w}_{x}^{\prime} X Y^{\prime} \hat{w}_{y}\right)}{\sqrt{E\left(\hat{w}_{x}^{\prime} X X^{\prime} \hat{w}_{x}^{\prime}\right) E\left(\hat{w}_{y}^{\prime} Y Y^{\prime} \hat{w}_{y}\right)}} \\
& =\frac{w_{x}^{\prime} C_{x y} w_{y}}{\sqrt{w_{x}^{\prime} C_{x x} w_{x} w_{y}^{\prime} C_{y y} w_{y}}}
\end{aligned}
$$

The maximum correlation is equal to the maximum of $\rho$, with respect to $w_{y}$ and $w_{x}$. The projections onto $w_{y}$ and $w_{x}$ are called canonical variates.

Canonical correlations are uncorrelated:

$$
\begin{aligned}
& E\left(x_{i} x_{j}\right)=E\left(w_{x i}^{\prime} X X^{\prime} w_{x j}\right)=w_{x i}^{\prime} C_{x x} w_{x j}=0 \\
& E\left(y_{i} y_{j}\right)=E\left(w_{y i}^{\prime} Y Y^{\prime} w_{y j}\right)=w_{y i}^{\prime} C_{y y} w_{y j}=0 \\
& E\left(x_{i} y_{j}\right)=E\left(w_{x i}^{\prime} X Y^{\prime} w_{y j}\right)=w_{x i}^{\prime} C_{x y} w_{y j}=0
\end{aligned} \quad \text { for } \quad i \neq j
$$

The total covariance matrix is a block matrix defined below, where $C_{x x}$ and $C_{y y}$ are within-sets covariance matrices of $\mathrm{X}$ and $\mathrm{Y}$ and $C_{x y}=C_{y x}^{\prime}$ is the between-sets covariance matrix.

$$
C=\left[\begin{array}{ll}
C_{x x} & C_{x y} \\
C_{y x} & C_{y y}
\end{array}\right]
$$

Eigenvalue $\rho^{2}$ corresponds to squared canonical correlation, and eigenvectors $\hat{w}_{x}, \hat{w}_{y}$ are the normalized canonical correlation basis vectors.

$$
\begin{aligned}
& C_{x x}^{-1} C_{x y} C_{y y}^{-1} C_{y x} \hat{w}_{x}=\rho^{2} \hat{w}_{x} \\
& C_{y y}^{-1} C_{y x} C_{x x}^{-1} C_{x y} \hat{w}_{y}=\rho^{2} \hat{w}_{y}
\end{aligned}
$$

Canonical correlations are obtained from the following equation;

$$
\begin{aligned}
& C_{x y} \hat{w}_{y}=\rho \lambda_{x} C_{x x} \hat{w}_{x} \\
& C_{y x} \hat{w}_{x}=\rho \lambda_{y} C_{y y} \hat{w}_{y}
\end{aligned}
$$

where

$$
\lambda_{x}=\lambda_{y}^{-1}=\sqrt{\frac{\hat{w}_{y}^{\prime} C_{y y} \hat{w}_{y}}{\hat{w}_{x}^{\prime} C_{x x} \hat{w}_{x}}}
$$

The statistical significance of the full canonical model can be found through Bartlett's test. $R^{2}$ represents the relationship between the two data sets:

$$
R^{2}=1-\Lambda
$$


$\Lambda$ expresses the unexplained variance in the model. By considering the obtained canonical functions, the term can be updated by the following (Pedhazur, 1997 p.939):

$\Lambda=\left(1-R_{c 1}^{2}\right)\left(1-R_{c 2}^{2}\right) \ldots\left(1-R_{c m}^{2}\right)$, where $R_{c 1}^{2}$ stands for the squared canonical correlation of the first canonical model. By convention, functions explaining a reasonable amount of variance, namely, a canonical correlation $R_{c}$ of more than 0.30, are preferred (Nimon \& Reio, 2011).

Standardized function coefficients and structure coefficients are prominent criteria for interpreting the canonical functions. Standardized function analogous to beta weights in regression correspond to weights assigned to observed variables after standardization, and structure coefficients are correlations between variables and a canonical variate (Sherry \&Henson, 2005).

However, Nimon, Henson, \& Gates (2010) emphasized that the examination of standardized function coefficients and structure coefficients may not provide sufficient information and that CCA findings do not provide a clear interpretation of a variable's unique or common contribution to a canonical effect. Nimon, Henson, \& Gates (2010) employed commonality analysis in addition to CCA. Their approach is based on partitioning the canonical variate and obtaining unique and common effects. Their study emphasizes that in the case of common effects, commonalities may be negative and this situation arises from suppressor variable effects. With the use of commonality analysis the effects of multicollinearity and suppression on the squared structure coefficients can be clearly seen.

The essential steps of CCA are summarized below (Nimon \& Reio, 2011):

-In the first stage, the number of canonical functions is determined by evaluating thesufficiency of shared variance $\left(R_{c}^{2}\right)$ of each canonical function.

-For the canonical variates of a given canonical set, there will be as many commonality coefficients as there are combinations of variables $\left(2^{\mathrm{k}}-1\right)$ in the other canonical set. Commonality analysis produces $\mathrm{k}$ unique effects and $2^{\mathrm{k}}-1-\mathrm{k}$ common effects. The commonalities are calculated according to formulas based on Mood's procedure. Nimon, Henson, \&Gates (2010) summarized the aforementioned procedure as below:

"In Mood's procedure, (1-x) was used to represent variables in the common variance subset and $x$ was used to represent variables not in the common variance subset. By negating the product of the variables in the subset and the variables not in the subset, deleting the -1 resulting from the expansion of the product, and replacing $x$ with $R^{2}$, Mood noted that the formula for computing any commonality coefficient can be derived" ( $p$. 459).

The commonality coefficient formulas for three variables are presented in Table 1. Nimon, Henson, \& Gates (2010) published a syntax of CCA to use with the R package.

Table 1. Unique and common formulas for decomposing a given canonical variate $(\mathrm{g})$ with three variables $(\mathrm{i}, \mathrm{j}, \mathrm{k})$ from the other canonical set (o)

$$
\begin{aligned}
& U_{(i)}=-\left(1-o_{i}\right) o_{j} o_{k}=R_{g[f n] . j j k}^{2}-R_{g[f n] . j k}^{2} \\
& U_{(j)}=-\left(1-o_{j}\right) o_{i} o_{k}=R_{g[f n] . i j k}^{2}-R_{g[f n] . i k}^{2} \\
& U_{(k)}=-\left(1-o_{k}\right) o_{i} o_{j}=R_{g[f n] . i j k}^{2}-R_{g[f n] . i j}^{2} \\
& C_{(i j)}=-\left(1-o_{i}\right)\left(1-o_{j}\right) o_{k}=R_{g[f n] . i k}^{2}+R_{g[f n] . j k}^{2}-R_{g[f n] . k}^{2}-R_{g[f n] . i j k}^{2} \\
& C_{(i k)}=-\left(1-o_{i}\right)\left(1-o_{k}\right) o_{j}=R_{g[f n] . i j}^{2}+R_{g[f n] . j k}^{2}-R_{g[f n] . j}^{2}-R_{g[f n] . i j k}^{2} \\
& C_{(j k)}=-\left(1-o_{i}\right)\left(1-o_{k}\right) o_{i}=R_{g[f n] . i j}^{2}+R_{g[f n] . i k}^{2}-R_{g[f n] . i}^{2}-R_{g[f n] . i j k}^{2} \\
& C_{(i j k)}=-\left(1-o_{i}\right)\left(1-o_{j}\right)\left(1-o_{k}\right) o_{i}=R_{g[f n] . i}^{2}+R_{g[f n] . j}^{2}-R_{g[f n] . k}^{2}-R_{g[f n] . i j}^{2}-R_{g[f n] . i k}^{2}-R_{g[f n] . j k}^{2}+R_{g[f n] . j k k}^{2}
\end{aligned}
$$

$\mathrm{f}(\mathrm{n})$ denotes canonical function.

Source: Nimon, Henson, \& Gates, 2010 p.711 
Commonality analysis decomposes $\mathrm{R}^{2}$ - type effect size into non-overlapping parts that describe both unique and common explanatory powers of sets of predictor variables in all their possible combinations. Partitioning the unique and commonly shared variance-shared variance helps determine the true predictive power of individual predictor variables and proves invaluable when determining parsimony in a multivariable model (Stellefson, Yannessa, \& Martel, 2012).

CCA can be considered a generalization of multiple linear regression analysis and multivariate analysis of variance (MANOVA). In MANOVA, one of the two sets consists of categorical variables, and regression analysis investigates the relationship between one dependent variable and a set of independent variables. Similarly, CCA seeks to determine the interrelationships between two sets of variables.

\section{Data and Findings}

In the present study, the relationship between the sub-indices of GII and the sub-indices of HPI were examined for 150 countries. As mentioned in Section 1, GII consists of maternal mortality rate (MM), adolescent birth rate (AB), female share of parliamentary seats (PS), female and male population with at least a secondary education (SC), and female and male labour-force participation rates (LF). However, we excluded the male population with at least a secondary education and male labour-force participation rates from the dataset due to a singularity problem. A singularity problem arises when including variables that are too highly correlated in a dataset. As required in discriminant analysis, the data matrix should be non-singular, which means that none of the variables can be a linear combination of other variables (we retained the variable with the highest correlation). Data was taken from the 2014 UNDP reports and the NEF database.

Table 2 presents descriptive statistics of the dataset. The standard deviation of variable MM is quite high. As already known, covariance matrices are sensitive to outlying observations and ignoring their existence may cause misleading interpretations. Accordingly, in most of the statistical analyses, outliers adversely affected findings from CCA. Before employing canonical correlation, outliers were detected by both classical and robust diagnostics. However, exclusion of outliers from the dataset did not change the significance of correlation coefficients or interpretations of our findings.

Table 2. Descriptive statistics of variables

\begin{tabular}{lllllllll}
\hline & LE & WB & FP & MM & AB & PS & SC & LF \\
\hline Min & 47.80 & 2.800 & 0.540 & 1.0 & 0.60 & 0.00 & 0.90 & 13.50 \\
Q1 & 64.40 & 4.425 & 1.405 & 11.5 & 12.62 & 12.70 & 27.20 & 44.73 \\
Median & 73.25 & 5.250 & 2.390 & 49.0 & 35.30 & 19.95 & 56.80 & 53.05 \\
Mean & 69.79 & 5.406 & 3.053 & 152.9 & 49.07 & 21.61 & 55.88 & 52.56 \\
Q3 & 76.83 & 6.375 & 4.362 & 200.0 & 73.60 & 28.35 & 85.22 & 61.95 \\
Max & 83.40 & 7.800 & 11.680 & 980.0 & 204.80 & 57.50 & 100.00 & 88.10 \\
Std & 9.83 & 1.17 & 2.16 & 208.44 & 43.32 & 11.40 & 31.12 & 16.43 \\
\hline
\end{tabular}

Table 3 presents correlations between the sub-indices of GII and HPI alongside the correlations between sub-indices of datasets. Confirming expectations, there are significant correlations between the two datasets. Correlations between LE and other variables except PS are significant; PS and FB, MM, and AB variables have insignificant correlations. Except the correlations between LF and variables WB, FB, and SC, the remaining relations are significant. 
Table 3. Correlations between HPI and GII variable sets

\begin{tabular}{|c|c|c|c|c|c|c|c|c|}
\hline & LE & WB & FP & MM & AB & PS & SC & LF \\
\hline$\overline{\mathbf{L E}}$ & 1,000 & $0,713^{*}$ & $0,609^{*}$ & $-0,838^{*}$ & $-0,737^{*}$ & 0,126 & $0,720^{*}$ & $-0,321^{*}$ \\
\hline WB & & 1,000 & $0,671^{*}$ & $-0,590^{*}$ & $-0,473^{*}$ & $0,280^{*}$ & $0,582^{*}$ & $-0,116$ \\
\hline FP & & & 1,000 & $-0,514^{*}$ & $-0,561^{*}$ & 0,096 & $0,611^{*}$ & $-0,079$ \\
\hline MM & & & & 1,000 & $0,772 *$ & $-0,089$ & $-0,724^{*}$ & $0,362^{*}$ \\
\hline $\mathbf{A B}$ & & & & & 1,000 & $-0,069$ & $-0,700^{*}$ & $0,305^{*}$ \\
\hline PS & & & & & & 1,000 & 0,103 & $0,238^{*}$ \\
\hline SC & & & & & & & 1,000 & $-0,104$ \\
\hline LF & & & & & & & & 1,000 \\
\hline
\end{tabular}

*Correlation is significant at the 0.01 level.

CCA concludes with three canonical functions since there are three variables in the smaller set. As seen in Table 4, the $\mathrm{R}_{\mathrm{c}}$ value of Function 1 is much larger than those of Functions 2 and 3. However, three of the functions are statistically significant according to $\chi^{2}$ statistics. Function 1 accounted for $86 \%\left(\mathrm{R}_{\mathrm{c}}{ }^{2}=0.858\right)$ of the variance between the two datasets.

Table 4. Canonical correlations between GII and HPI

\begin{tabular}{lllrll}
$R_{c}$ & $R_{c}^{2}$ & Wilks' s $\Lambda$ & $\chi^{2}$ & df & Sig. \\
\hline 0.921 & 0.858 & 0.119 & 219.56 & 15 & 0 \\
0.342 & 0.117 & 0.721 & 32.277 & 8 & 0 \\
0.323 & 0.104 & 0.882 & 11.772 & 3 & 0.00078 \\
\hline
\end{tabular}

To interpret the canonical variables, standardized function coefficients were used (Table 5). Function 1 (HPI) shows that LE has more effect (-0.98) compared to variables WE (0.298) and FT (-0.168). Similarly, the squared structure coefficients of Function 1 indicate LE, WE, and FT explain 96.7\%, 38.2\%, and 41.7\% of the variance, respectively (Table 6). This finding reveals that a part of the variance is commonly explained by LE, WE, and FT because their individual contributions are more than $100 \%$ of variance in the canonical variate. Function 1 (GII) indicates that MM has the highest effect $(0.631)$ and contribution to variance $(94.7 \%)$ to the canonical variate. $\mathrm{AB}$ has the second highest contribution to variance $(72.8 \%)$.

Table 5. Standardized canonical coefficients

\begin{tabular}{llllrrrr}
\hline GII & CV 1 & CV 2 & CV 3 & HPI & CV 1 & CV 2 & CV 3 \\
\hline MM & 0.631 & 0,753 & 0.515 & LE & -0.985 & -0.194 & -0.925 \\
AB & 0.285 & -0.997 & 0.283 & WE & 0.298 & -0.991 & 0.898 \\
PS & -0.098 & -0.258 & 0.712 & FT & -0.168 & 0.929 & 0.461 \\
SC & $-0,179$ & 0.206 & 0.842 & & & & \\
LF & 0.092 & 0,109 & 0.241 & & & & \\
\hline
\end{tabular}

PS (-0.098) and SC (-0.179) have negative coefficients (Table 5) and different effects from the remaining canonical covariates. Total variance is again more than $100 \%$, which reveals the need for clear interpretation. To eliminate ambiguity in interpretation, commonality analysis was also employed. Table 7 indicates the partitioning of the GII components variate by the variables in the HPI set. 
Table 6. Squared structure coefficients

\begin{tabular}{llllrlrl}
\hline GII & CV 1 & CV 2 & CV 3 & HPI & CV 1 & CV 2 & CV 3 \\
\hline MM & 0,947 & 0,012 & 0,035 & LE & 0,967 & 0,01 & 0,004 \\
AB & 0,728 & 0,12 & 0,009 & WE & 0,382 & 0,135 & 0,384 \\
PS & 0,073 & 0,071 & 0,092 & FT & 0,417 & 0,235 & 0,319 \\
SC & 0,645 & 0,003 & 0,227 & & & & \\
LF & 0,161 & 0,011 & 0,39 & & & & \\
\hline
\end{tabular}

GII components variate is mostly explained by variance unique to LE, namely $52.11 \%$ of the canonical effect which is consistent with its larger standardized function coefficient (Table 5). The second largest contribution to variance is from common to LE, WE, and FT at $32.48 \%$. Approximately $43.47 \%$ of the canonical effect arises from multicolinearity between LE, WE, and FT.

Table 7. Partitioning of Function I's GII components canonical variate

\begin{tabular}{lll}
\hline & Coefficient & \% Total \\
\hline Unique to LE & 0.4397 & 52.11 \\
Unique to WE & 0.0298 & 3.53 \\
Unique to FT & 0.0126 & 1.5 \\
Common to LE, and WE & 0.024 & 2.15 \\
Common to LE, and FT & 0.0738 & 8.74 \\
Common to WE, and FT & 0.0062 & 0.1 \\
Common to LE, WE, and FT & 0.274 & 32.48 \\
Total & 0.8437 & 100 \\
\hline
\end{tabular}

Similarly, Table 8 shows the partitioning of the HPI variate by the GII components. The highest amount of common explained variance is from $\mathrm{MM}, \mathrm{AB}$ and $\mathrm{SC}$, at $43.88 \%$ of the canonical effect. Considering this common variance, choosing one of these sub-indicators in the model may provide more accurate results. It should be noted that approximately $87 \%$ of the canonical effect arises from multicolinearity. The highest unique contribution is $10.73 \%$ from MM, followed by contributions from AB, PS, and LF. However, LF seems to have a suppressing effect. As seen in Table 8, there are some negative common commonality coefficients, which indicates the possible presence of a suppressing effect of LF. The amount of variance in a canonical variate is confounded by a set of variables (Nimon, Henson, \& Gates, 2010). MM, AB, and PS uniquely accounted for $10.73 \%, 2.38 \%$, and $0.88 \%$ of the canonical effect, respectively, only after they were scrubbed of the irrelevant variance in common with LF (Nimon and Reio, 2011). The standardized coefficient of LF has the same direction of effect with MM and AB; this contradiction can be thought as an indicator of the suppressor effect. 
Table 8. Partitioning of Function I's HPI components canonical variate

\begin{tabular}{|c|c|c|}
\hline & Coefficient & $\begin{array}{c}\% \\
\text { t Total }\end{array}$ \\
\hline Unique to MM & 0.0905 & 10.73 \\
\hline Unique to $A B$ & 0.0201 & 2.38 \\
\hline Unique to PS & 0.0074 & 0.88 \\
\hline Unique to SC & 0.0035 & 0.42 \\
\hline Unique to LF & 0.0052 & 0.61 \\
\hline Common to MM, and AB & 0.0867 & 10.28 \\
\hline Common to MM, and PS & 0.0136 & 1.61 \\
\hline Common to $\mathrm{AB}$, and PS & 0.0002 & 0.02 \\
\hline Common to MM, and SC & 0.0320 & 3.8 \\
\hline Common to $\mathrm{AB}$, and $\mathrm{SC}$ & 0.0136 & 1.61 \\
\hline Common to PS, and SC & 0.0004 & 0.05 \\
\hline Common to MM, and LF & 0.0262 & 3.11 \\
\hline Common to $\mathrm{AB}$, and $\mathrm{LF}$ & 0.0042 & 0.49 \\
\hline Common to PS, and LF & -0.0023 & -0.28 \\
\hline Common to SC, and LF & -0.0020 & -0.24 \\
\hline Common to MM, $\mathrm{AB}$, and PS & 0.0122 & 1.45 \\
\hline Common to $\mathrm{MM}, \mathrm{AB}$, and $\mathrm{SC}$ & 0.3702 & 43.88 \\
\hline Common to MM, PS, and SC & 0.0022 & 0.26 \\
\hline Common to AB, PS, and SC & 0.0005 & 0.06 \\
\hline Common to $\mathrm{MM}, \mathrm{AB}$, and $\mathrm{LF}$ & 0.0716 & 8.49 \\
\hline Common to MM, PS, and LF & -0.0057 & -0.68 \\
\hline Common to AB, PS, and LF & -0.0009 & -0.1 \\
\hline Common to MM, SC, and LF & -0.0096 & -1.14 \\
\hline Common to $\mathrm{AB}, \mathrm{SC}$, and $\mathrm{LF}$ & -0.0032 & -0.38 \\
\hline Common to PS, SC, and LF & 0.0006 & 0.07 \\
\hline Common to MM, AB, PS, and SC & 0.0552 & 6.55 \\
\hline Common to MM, AB, PS, and LF & -0.0068 & -0.81 \\
\hline Common to MM, AB, SC, and $\mathrm{LF}$ & 0.0747 & 8.86 \\
\hline Common to MM, PS, SC, and LF & 0.0004 & 0.05 \\
\hline Common to AB, PS, SC, and LF & 0.0007 & 0.08 \\
\hline Common to MM, AB, PS, SC, and $\mathrm{LF}$ & -0.0158 & -1.87 \\
\hline Total & 0.8437 & 100 \\
\hline
\end{tabular}

\section{Conclusion}

This study investigates the relationship between the sub-indices of HPI and GII by canonical commonality analysis. Although there are various studies on the relation between gender equality and happiness, they have not considered gender inequality on the grounds of reproductive health, empowerment, and economic status. On the other hand, to the best of our knowledge, no previous studies have employed a canonical commonality approach to evaluate the relationship between happiness and gender inequality. Using commonality approach helps reveal the previously undiscovered effects of multicolinearity and suppression. 
CCA resulted in three canonical functions; however, the first canonical function explained $86 \%$ of the variance between GII and HPI, which indicates a strong correlation between two datasets. Since CCA ignores the interaction of subindicators which may affect the validity of findings, the study focused on both the unique and common effects of each sub-indicator in the model. Findings reveal that approximately $32 \%$ of the canonical effect of GII components variate is explained by common variance from LE, WE, and FT. The highest unique contribution is from LE, which explains the $52 \%$ of the canonical effect. Additionally, $43 \%$ of the canonical effect arises from the multicolinearity between WE, LE, and FT.

Similarly, HPI components variate is mostly explained by the common variance. MM, AB and SC together explain $44 \%$ of the variance of the canonical effect. Therefore, choosing one of these sub-indicators may provide more accurate results. $\mathrm{LF}$ is a suppressing variable. $\mathrm{MM}, \mathrm{AB}$, and PS uniquely accounted for $10.73 \%, 2.38 \%$, and $0.88 \%$ of the canonical effect, respectively, only after they were scrubbed of the irrelevant variance they had in common with LF. The standardized coefficient of LF has the same direction of effect with MM and AB. This contradiction arises from the suppressing effect. It should be noted that if this study had considered only CCA, the question of why an increase in the share of LF yielded a decrease in happiness would be unanswered.

As a result, regardless of the contribution of each sub-indicators' unique and common effects, just examining the standardized canonical covariates to evaluate the relative importance of sub-indicators may cause misinterpretation.

\section{References}

Blanchflower, D. G., \& Oswald, A. J. (2004). Well-being over Time inBritain and the USA. Journal of Public Economics, 88, 1359-1386. http://dx.doi.org/10.1016/S0047-2727(02)00168-8

Borga, M. (2001). Canonical Correlation a Tutorial. Retrieved from https://www.cs.cmu.edu/ tom/10701_sp11/slides/CCA_tutorial.pdf

Cesare, M. C., \& Amori, A. (2006). Gender and happiness in Italy. Paper presented at the 2006 meeting of the population association of America annual meeting, Los Angeles, California. Retrieved from http://paa2006.princeton.edu/abstractViewer.aspx?submissionId=60246)

Cho, R. (2015). Why Happiness Is Important? Retrieved from http://blogs.ei.columbia.edu/2015/04/23/why-happiness-is-important/

Clark, A., \& Oswald, A. J. (1994). Unhappiness and Unemployment. Economic Journal, 104, 648-659. http://dx.doi.org/10.2307/2234639

Diener, E., \& Seligman, M. E. P. (2004). Beyond money: Toward an economy of well-being. Psychological Science in the Public Interest, 5, 1-31. http://dx.doi.org/10.1111/j.0963-7214.2004.00501001.x

Diener, E., Sandvik, E., Seidlitz, L., \& Diener, M. (1993). The relationship between income and subjective well-being: Relative or absolute? Social Indicators Research, 28, 195-223. http://dx.doi.org/10.1007/BF01079018

Easterlin R. A. (2001). Income and happiness: towards a unified theory. Economic Journal, 111, 465-484. http://dx.doi.org/10.1111/1468-0297.00646

Hotelling, H. (1936). Relations between two sets of variants. Biometrika, 28, 321-377. http://dx.doi.org/10.1093/biomet/28.3-4.321

Huppert, F. A., \& Whittington, J. E. (2003). Evidence for the independence of positive and negative wellbeing: Implications for quality of life assessment. British Journal of Health Psychology, 8(1), 107-122. http://dx.doi.org/10.1348/135910703762879246

Kahneman, D., \& Deaton, A. (2010). High income improves evaluation of life but not emotional well-being. Proceedings of the National Academy of Sciences of the USA, 107, 16489-16493. http://dx.doi.org/10.1073/pnas.1011492107

Marks, N., Abdallah, S., Simms A., \& Thompson S. (2006). The Happy Planet Index. New Economics Foundation, London.

McGarigal, K., Cushman, S. A., \& Stafford, S. (2000). Multivariate statistics for wildlife and ecology research. Springer-Verlag, New York, New York, USA. http://dx.doi.org/10.1007/978-1-4612-1288-1

New Economic Foundation. (2012). The Happy Planet Index: 2012 Report. Retrieved from http://www.happyplanetindex.org/assets/happy-planet-index-report.pdf 
Nimon, K., \& Reio T. (2011). The use of Canonical Commonality Analysis for Quantitative Theory Building. Human Resource Development Review, 10(4), 451-463. http://dx.doi.org/10.1177/1534484311417682

Nimon, K., Henson, R., \& Gates, M. (2010). Revisiting Intepretation of Canonical Correlation Analysis: A Tutorial and Demonstration of Canonical commonality Analysis. Multivariate Behavioral Research, 45, 702-724. http://dx.doi.org/10.1080/00273171.2010.498293

Pedhazur, E. J. (1997). Multiple regression in behavioral research (3rd ed.). Orlando, FL: Harcourt Brace.

Sen, A. (2005). Human Rights and Capabilities. Journal of Human Development and Capabilities, 6(2), 151-166. http://dx.doi.org/10.1080/14649880500120491

Sherry, A., \& Henson, R. K. (2005). Conducting and interpreating canonical correlation analysis in personality research: A user friendly primer. Journal of Personlity Assesment, 84, 37-48. http://dx.doi.org/10.1207/s15327752jpa8401_09

Stellefson, M., Yannessa J. F., \& Martel, G. F. (2012). Using canonical commonality analysis to examine the predictive quality of aging and falls efficacy on balance functioning in older adults. Eval Health Prof., 35(2), 239-255. http://dx.doi.org/10.1177/0163278711403925

Tiefenbach, T., \& Kohlbacher, F. (2013). Happiness from the viewpoint of Economics: Findings from Recent Survey Data in Japan (DIJ Working Paper 13/1). Retrieved from http://www.dijtokyo.org/publications/WP1301_Tiefenbach_Kohlbacher.pdf

United Nations. (2015). United Nations Development Report, 2014. Retrieved from http://hdr.undp.org/sites/default/files/hdr14-report-en-1.pdf

Van Praag, B. M. S., \& Ferrer-i-Carbonell, A. (2008). Happiness Quantified: A Satisfaction Calculus Approach. Oxford: UK. Revised \& Paperback edition: Oxford University Press.

Veenhoven, R. (1994). Is happiness a trait? Social Indicators Research, 32, 101-160. http://dx.doi.org/10.1007/BF01078732 